10,11

\title{
О вычислении температуры Дебая и температуры фазового перехода кристалл-жидкость для бинарного сплава замещения
}

\author{
(C) М.Н. Магомедов
}

Институт проблем геотермии ДагНЦ РАН, Махачкала, Россия

E-mail: mahmag4@mail.ru

(Поступила в Редакцию 16 августа 2017 г.

В окончательной редакции 30 октября 2017 г.)

\begin{abstract}
Предложена методика получения параметров межатомного потенциала парного взаимодействия для бинарного сплава замещения, которая учитывает отклонение параметра решетки сплава от правила Вегарда. На основе данной методики сделан расчет температуры Дебая и параметров Грюнайзена для сплава SiGe. Показано, что все эти функции изменяются при изменении концентрации германия нелинейно. На основании данной методики и критерия плавления Линдеманна предложена методика расчета температур солидуса и ликвидуса для неупорядоченного сплава замещения. Методика протестирована на сплаве $\mathrm{SiGe}$ и показала хорошее согласие с экспериментальными данными. Показано, что при уменьшении размера нанокристалла твердого раствора замещения разница между температурами ликвидуса и солидуса уменьшатся тем больше, чем заметнее форма нанокристалла отклонена от наиболее энергетически оптимальной формы.
\end{abstract}

Работа выполнена при финансовой поддержке РФФИ в рамках научного проекта № 16-03-00041_а и Программы Президиума РАН (программа № I.13).

DOI: 10.21883/FTT.2018.05.45797.251

\section{1. Введение}

На сегодняшний день нет единого мнения о том, как рассчитать температуру Дебая $(\Theta)$ для сплава бинарного состава $\mathrm{A}_{1-C} \mathrm{~B}_{C}$ по параметрам чистых компонентов. Поэтому используют различные процедуры усреднения. Чаще всего используется выражение среднего арифметического по концентрации [1], т. е.

$$
\Theta\left(\mathrm{A}_{1-C} \mathrm{~B}_{C}\right)=(1-C) \Theta_{\mathrm{A}}+C \Theta_{\mathrm{B}}=\Theta_{\mathrm{A}}+C\left(\Theta_{\mathrm{B}}-\Theta_{\mathrm{A}}\right),
$$

где $\Theta_{\mathrm{A}}$ - температура Дебая кристалла растворителя, $\Theta_{\mathrm{B}}$ - температура Дебая кристалла растворенного элемента, $C$ - атомная концентрация растворенного элемента.

Вместе с тем как было экспериментально показано [2], зависимость $\Theta(C)$ в общем случае имеет нелинейный характер. Известно, что температура Дебая зависит от плотности, а плотность в подавляющем большинстве твердых сплавов меняется с концентрацией нелинейно. Возникает вопрос - как будет зависеть температура Дебая от концентрации бинарного сплава, если его параметр решетки меняется с концентрацией нелинейно? Для ответа на поставленный вопрос рассмотрим сплав двух изоструктурных веществ А и В и используем приближение „взаимодействия только ближайших соседей“, которое также называют „квазихимическим“ приближением [3]. При изучении бинарной смеси $\mathrm{A}_{1-C} \mathrm{~B}_{C}$ сначала необходимо определить закон парного межатомного взаимодействия как однородных атомов (А-А и В-В), так и разных атомов между собой: (А-В).

\section{2. Межатомный потенциал для бинарного сплава}

Представим парное межатомное взаимодействие в виде четырехпараметрического потенциала Ми-Леннард-Джонса, имеющего вид [4]

$$
\varphi(r)=\frac{D}{(b-a)}\left[a\left(\frac{r_{0}}{r}\right)^{b}-b\left(\frac{r_{0}}{r}\right)^{a}\right],
$$

где $D$ и $r_{0}$ - глубина и координата минимума потенциала, $b>a>1$ - параметры.

Вопрос о том, как из параметров потенциала (2) для чистых компонентов получить параметры межатомного потенциала для пары разнородных атомов до сих пор не решен. Поэтому во многих работах используются правила комбинирования Лоренца-Бертло (Lorentz-Berthelot combining rules), в которых для получения $r_{0}$ и $D$ для разных атомов используется среднее арифметическое и среднее геометрическое от соответствующих параметров чистых веществ [4, стр. 149, 185, 447]

$$
\begin{gathered}
r_{0 \mathrm{AB}}=\left(r_{0 \mathrm{~A}}+r_{0 \mathrm{~B}}\right) / 2, \\
D_{\mathrm{AB}}=\left(D_{\mathrm{A}} D_{\mathrm{B}}\right)^{1 / 2} .
\end{gathered}
$$

Что касается степенных параметров потенциала (2), то для них до сих пор нет единого мнения по процедуре усреднения как для $a$, так и для $b$. Поэтому для простоты берут среднее арифметическое от соответствующих значений для чистых веществ.

Выражения (3) и (4) первоначально были предложены для изучения смеси химически не взаимодействующих 
газов: (3) было предложено Н.A. Lorentz в 1881 году, a (4) ввел Daniel Berthelot в 1898 году. Выражение (3) противоречит правилу аддитивности молярных объемов, и не учитывает того факта, что в большинстве твердых сплавов параметр решетки меняется с концентрацией нелинейно, т.е. наблюдается отклонение от правила Вегарда (Vegard's law) [5,6]. Формула (4) также ничем не обоснована. Но, несмотря на это, выражения (3) и (4) до сих пор используются для расчета свойств бинарных смесей газов, жидкостей и твердых сплавов различной природы.

Для усреднения параметров потенциала (2) мы используем процедуру, которая учитывает отклонение параметра решетки сплава от правила Вегарда. Пусть $P_{\mathrm{A}}$ и $P_{\mathrm{B}}$ это вероятности нахождения в узле смешанной решетки атома сорта А и В соответственно. Тогда для вероятности одновременного нахождения в соседних узлах решетки атомов одного сорта и атомов разных сортов можно принять: $P_{\mathrm{AA}}=P_{\mathrm{A}}^{2}, P_{\mathrm{BB}}=P_{\mathrm{B}}^{2}$ и $P_{\mathrm{AB}}=2 P_{\mathrm{A}} P_{\mathrm{B}}$. Число парных связей для пар атомов одного сорта и для пар разных атомов равно [3]

$$
\begin{gathered}
N_{\mathrm{AA}}=\left(k_{n} / 2\right) N P_{\mathrm{A}}^{2}, \quad N_{\mathrm{BB}}=\left(k_{n} / 2\right) N P_{\mathrm{B}}^{2}, \\
N_{\mathrm{AB}}=k_{n} N P_{\mathrm{A}} P_{\mathrm{B}},
\end{gathered}
$$

где $N=N_{\mathrm{A}}+N_{\mathrm{B}}-$ общее число атомов в сплаве, $k_{n}-$ первое координационное число, т.е. число ближайших к данному атому узлов решетки.

Тогда, учитывая, что общее число парных связей в сплаве равно: $N N=\left(k_{n} / 2\right) N$, для среднего значения какого-либо $H$-параметра межатомного потенциала (2) сплава можно получить выражение

$$
H\left(\mathrm{~A}_{1-C} \mathrm{~B}_{C}\right)=P_{\mathrm{A}}^{2} H_{\mathrm{A}}+P_{\mathrm{B}}^{2} H_{\mathrm{B}}+2 P_{\mathrm{A}} P_{\mathrm{B}} H_{\mathrm{AB}},
$$

где $H_{\mathrm{AB}}$ - параметр межатомного потенциала (2) для решетки с равным числом однородно распределенных по решетке атомов обеих сортов, с учетом релаксации такой решетки к минимуму энергии.

Входящие в (5) функции $P_{\mathrm{A}}$ и $P_{\mathrm{B}}=1-P_{\mathrm{A}}$ определим как геометрические вероятности того, что точка, случайно поставленная на линию длиной $(1-C) r_{0 \mathrm{~A}}+C r_{0 \mathrm{~B}}$, попадет на отрезки $r_{0 \mathrm{~A}}$ или $r_{0 \mathrm{~B}}$ соответственно

$$
\begin{aligned}
& P_{\mathrm{A}}(C)=\frac{(1-C) r_{0 \mathrm{~A}}}{(1-C) r_{0 \mathrm{~A}}+C r_{0 \mathrm{~B}}}, \\
& P_{\mathrm{B}}(C)=\frac{C r_{0 \mathrm{~B}}}{(1-C) r_{0 \mathrm{~A}}+C r_{0 \mathrm{~B}}} .
\end{aligned}
$$

При внесении атомов одного сорта в решетку из атомов другого сорта объем общей решетки $V_{\mathrm{AB}}$ релаксирует так, чтобы достигался минимум свободной энергии. Поэтому разложим удельную (на атом своей подрешетки) свободную энергию для каждой из подрешеток в ряд по степеням отклонения удельного объема:
$\Delta V_{i}=V_{\mathrm{AB}}-V_{i 0}$, при постоянной температуре $(T)$

$$
\begin{aligned}
F(\mathrm{~A})=F(\mathrm{~A})_{0} & +\left(\frac{\partial F(\mathrm{~A})}{\partial V}\right)_{T, V_{\mathrm{A}}} \Delta V_{\mathrm{A}} \\
& +\frac{1}{2}\left(\frac{\partial^{2} F(\mathrm{~A})}{\partial V^{2}}\right)_{T, V_{\mathrm{A}}}\left(\Delta V_{\mathrm{A}}\right)^{2}, \\
F(\mathrm{~B})=F(\mathrm{~B})_{0} & +\left(\frac{\partial F(\mathrm{~B})}{\partial V}\right)_{T, V_{\mathrm{B}}} \Delta V_{\mathrm{B}} \\
& +\frac{1}{2}\left(\frac{\partial^{2} F(\mathrm{~B})}{\partial V^{2}}\right)_{T, V_{\mathrm{B}}}\left(\Delta V_{\mathrm{B}}\right)^{2} .
\end{aligned}
$$

Таким образом, добавочная удельная свободная энергия решетки с равным числом атомов разного сорта будет равна

$$
\Delta F_{\mathrm{AB}}=-P\left(\Delta_{\mathrm{A}}+\Delta V_{\mathrm{B}}\right)+\frac{1}{2}\left[\frac{B_{\mathrm{A}}}{V_{\mathrm{A}}}\left(\Delta V_{\mathrm{A}}\right)^{2}+\frac{B_{\mathrm{B}}}{V_{\mathrm{B}}}\left(\Delta V_{\mathrm{B}}\right)^{2}\right],
$$

где

$$
P=-(\partial F(\mathrm{~A}) / \partial V)_{T}=-(\partial F(\mathrm{~B}) / \partial V)_{T}
$$

- давление, которое одинаково для обеих решеток,

$$
B_{i}=V_{i}\left(\partial^{2} F(i) / \partial V^{2}\right)_{T}=-V_{i}\left(\partial P / \partial V_{i}\right)_{T}
$$

- изотермический модуль упругости для решетки $i$-го компонента.

Из условия минимума функции (7):

$$
\left(\partial \Delta F_{\mathrm{AB}} / \partial V_{\mathrm{AB}}\right)_{T, P}=0,
$$

можно получить выражение для удельного объема смешанной решетки, состоящей из равного числа атомов разных сортов (т.е. для решетки эквиатомного состава), которая находится под давлением

$$
V_{\mathrm{AB}}=\frac{V_{\mathrm{A}}+C_{N} V_{\mathrm{B}}}{1+C_{N}}+2 \frac{P V_{\mathrm{A}}}{B_{\mathrm{A}}\left(1+C_{N}\right)},
$$

где введена функция, учитывающая разницу в сжимаемостях чистых компонент

$$
C_{N}=\frac{B_{\mathrm{B}}}{B_{\mathrm{A}}} \frac{V_{\mathrm{A}}}{V_{\mathrm{B}}} .
$$

Так как исходные и смешанная решетки имеют одинаковую структуру, то для параметра $r_{0 \text { Ав }}$ смешанной решетки эквиатомного состава, находящейся при нулевых значениях давления и температуры $(P=0, T=0 \mathrm{~K})$ можно принять

$$
r_{0 \mathrm{AB}}=\left(\frac{r_{0 \mathrm{~A}}^{3}+C_{N} r_{0 \mathrm{~B}}^{3}}{1+C_{N}}\right)^{1 / 3},
$$

где параметр, учитывающий разницу в сжимаемостях чистых компонент для смешанной решетки эквиатомного состава, будет иметь вид

$$
C_{N}=\frac{R_{\mathrm{B}}}{B_{\mathrm{A}}}\left(\frac{r_{0 \mathrm{~A}}}{r_{0 \mathrm{~B}}}\right)^{3} .
$$


Для простоты расчет параметра $D_{\text {Ав }}$ будем проводить с помощью выражения (4), а для расчета величин $b_{\mathrm{AB}}$ и $a_{\mathrm{AB}}$ используем среднее арифметическое от соответствующих значений для чистых компонент

$$
\begin{gathered}
D_{\mathrm{AB}}=\left(D_{\mathrm{A}} D_{\mathrm{B}}\right)^{1 / 2}, b_{\mathrm{AB}}=\left(b_{\mathrm{A}}+b_{\mathrm{B}}\right) / 2, \\
a_{\mathrm{AB}}=\left(a_{\mathrm{A}}+a_{\mathrm{B}}\right) / 2 .
\end{gathered}
$$

Значение средней массы атома для сплава $m(C)$ будем рассчитывать, как среднее геометрическое от масс составляющих сплав атомов

$$
m(C)=\left(\frac{P_{\mathrm{A}}}{m_{\mathrm{A}}}+\frac{P_{\mathrm{B}}}{m_{\mathrm{B}}}\right)^{-1} .
$$

Таким образом, мы моделируем твердый раствор $\mathrm{A}_{1-C} \mathrm{~B}_{C}$ из $N_{\mathrm{A}}$ и $N_{\mathrm{B}}$ атомов с разной атомной массой изоструктурным виртуальным кристаллом из $N=N_{\mathrm{A}}+N_{\mathrm{B}}$ одинаковых атомов, масса каждого из которых меняется с концентрацией $\left(C=N_{\mathrm{B}} / N\right)$ по формуле (13), а параметры парного межатомного взаимодействия меняются с $C$ согласно соотношениям (5), (6) и $(10)-(12)$.

\section{3. Методика расчета температуры Дебая, параметров Грюнайзена и фазовой диаграммы раствора}

Получив зависимость параметров потенциала (2) и средней массы атома от концентрации компонентов, можно рассчитать температуру Дебая для сплава по методике, предложенной в $[7,8]$ для однокомпонентного кристалла

$$
\Theta(m)=A_{m}(m) \xi\left[-1+\left(1+\frac{8 D}{k_{\mathrm{B}} A_{w}(m) \xi^{2}}\right)^{1 / 2}\right],
$$

где $k_{\mathrm{B}}$ - постоянная Больцмана, функция $A_{w}(m)$ возникает из-за учета энергии „нулевых колебанийс атомов в кристалле:

$$
\begin{gathered}
A_{w}(m)=K_{R}(m) \frac{5 k_{n} a b(b+1)}{144(b-a)}\left(\frac{r_{0}}{c}\right)^{b+2}, \\
K_{R}(m)=\frac{\hbar^{2}}{k_{\mathrm{B}} r_{0}^{2} m}, \quad \xi=\frac{9}{k_{n}},
\end{gathered}
$$

где $\hbar-$ постоянная Планка, $c-$ расстояние между центрами ближайших атомов.

Исходя из (14) и (15) можно рассчитать первый $(\gamma)$, второй $(q)$ и третий $(z)$ параметры Грюнайзена по формулам

$$
\begin{gathered}
\gamma=-\left(\frac{\partial \ln \Theta}{\partial \ln V}\right)_{T}=\frac{b+2}{6\left(1+X_{w}\right)}, \\
g=\left(\frac{\partial \ln \gamma}{\partial \ln V}\right)_{T}=\gamma \frac{X_{w}\left(1+2 X_{w}\right)}{\left(1+X_{w}\right)},
\end{gathered}
$$

$$
\begin{aligned}
z & =-\left(\frac{\partial \ln q}{\partial \ln V}\right)_{T}=\gamma\left(1+4 X_{w}\right)-2 q \\
& =\gamma\left(\frac{1+3 X_{w}}{1+X_{w}}\right)=\frac{(b+2)}{6} \frac{\left(1+3 X_{w}\right)}{\left(1+X_{w}\right)^{2}},
\end{aligned}
$$

где $X_{w}=A_{w}(m) \xi / \Theta-$ величина, определяющая роль „нулевых колебаний“ атомов при расчете температуры Дебая и параметров Грюнайзена.

С помощью (14)-(18) можно рассчитать как температуру Дебая и параметры Грюнайзена сплава: $\Theta(m)$, $\gamma(m), q(m), z(m)$, так и парциальные значения данных параметров для составляющих сплав компонентов: $\Theta\left(m_{i}\right), \gamma\left(m_{i}\right), q\left(m_{i}\right), z\left(m_{i}\right)$, т.е. для подрешетки атомов массой $m_{i}$, в которой все параметры межатомного потенциала меняются с концентрацией по предложенным здесь формулам (5), (6) и (10)-(12).

Исходя из модели плавления Линдеманна, для температуры плавления однокомпонентного вещества было получено выражение [9]

$$
k_{\mathrm{B}} T_{m}=\left(\frac{3}{16}\right) X_{L}^{2} m\left(\frac{c k_{\mathrm{B}} \Theta}{\hbar}\right)^{2},
$$

где $X_{L}=\left(\left\langle r^{2}\right\rangle / c^{2}\right)^{1 / 2}-$ параметр Линдеманна, представляющий собой отношение амплитуды колебания атомов к $c$ - расстоянию между центрами ближайших атомов при температуре плавления.

Известно, что при нагреве твердого раствора сначала плавится подрешетка более легкоплавкого компонента B, а при охлаждении жидкого сплава сперва кристаллизуется подрешетка более тугоплавкого компонента А. Исходя из этого и формулы (19) для $T_{s}$ - температуры солидуса (для плавления подрешетки более легкоплавкого компонента В) и $T_{l}-$ температуры ликвидуса (для кристаллизации более тугоплавкого компонента А) можно принять выражения следующего вида:

$$
\begin{aligned}
T_{s}(C)= & T_{m}(\mathrm{~B}) P_{\mathrm{B}}(C) \frac{m(C)}{m_{\mathrm{B}}}\left[\frac{c(C) \Theta(m)}{c_{\mathrm{B}} \Theta\left(m_{\mathrm{B}}\right)}\right]^{2}\left[\frac{X_{L \mathrm{~B}}}{X_{L}(C)}\right]^{2} \\
& +T_{m}(\mathrm{~A}) P_{\mathrm{A}}(C), \\
T_{l}(C)= & T_{m}(\mathrm{~A}) P_{\mathrm{A}}(C) \frac{m(C)}{m_{\mathrm{A}}}\left[\frac{c(C) \Theta(m)}{c_{\mathrm{A}} \Theta\left(m_{\mathrm{A}}\right)}\right]^{2}\left[\frac{X_{L \mathrm{~A}}}{X_{L}(C)}\right]^{2} \\
& +T_{m}(\mathrm{~B}) P_{\mathrm{B}}(C) .
\end{aligned}
$$

Здесь $T_{m}(i)$ - температура плавления чистой решетки для $i$-го компонента, $c(C) / c_{i}$ - отношение расстояний между центрами ближайших атомов в решетке раствора и в чистой решетке $i$-го компонента, $\Theta(m) / \Theta\left(m_{i}\right)-$ отношение температуры Дебая в решетке раствора и парциальной температуры Дебая для подрешетки $i$-го компонента, $X_{L}(C) / X_{L i}$ - отношение параметров Линдеманна в решетке раствора и в чистой решетке $i$-го компонента.

Отметим, что на сегодняшний день в литературе нет выражений типа (20) и (21), позволяющих рассчитать 
Таблица 1. Параметры межатомного потенциала (2), определенные в $[8,10]$, экспериментальные значения модуля упругости из [11] и температуры плавления из [12], и рассчитанные в [13] значения параметра Линдеманна

\begin{tabular}{c|c|c|c|c|c|c|c|c}
\hline Вещество & $m, \mathrm{amu}$ & $r_{0}, 10^{-10} \mathrm{~m}$ & $D / k_{\mathrm{B}}, \mathrm{K}$ & $b$ & $a$ & $B, \mathrm{GPa}[10,11]$ & $T_{m}, \mathrm{~K}[12]$ & $X_{L}[13]$ \\
\hline \multirow{2}{*}{$\mathrm{Si}$} & 28.09 & 2.351 & $\begin{array}{l}64286.16 \\
26921.28\end{array}$ & 4.0 & 2.48 & 97.7 & 1687.15 & $0.136 \pm 0.015$ \\
$\mathrm{Ge}$ & 72.59 & 2.450 & $\begin{array}{l}46764.12 \\
22511.76\end{array}$ & 4.3 & 2.75 & 74.9 & 1211.45 & $0.1245 \pm 0.015$
\end{tabular}

Примечание. В колонке для $D / k_{\mathrm{B}}$ в первой строке показано значение $D_{b} / k_{\mathrm{B}}$, а во второй строке $D_{s} / k_{\mathrm{B}}$.

функции $T_{S}(C)$ и $T_{l}(C)$ по параметрам парного межатомного потенциала чистых компонент.

\section{4. Результаты расчета для твердого раствора SiGe}

Для расчетов был выбран сплав замещения $\mathrm{SiGe}$, который на всем интервале концентрации имеет структуру алмаза: $k_{n}=4$. Параметры парного межатомного потенциала (2) для чистых кристаллов $\mathrm{Si}$ и $\mathrm{Ge}$ были определены в $[8,10]$ и представлены в табл. 1. Там же показаны экспериментальные значения модуля упругости из [11] и температуры плавления из [12], а также рассчитанные в [13] значения параметра Линдеманна для кремния и германия.

Как было показано в $[8,10,14]$, значение глубины потенциальной ямы для ковалентных кристаллов можно определить двумя путями: из модуля упругости $\left(B_{0}\right)$ и из удельной энергии атомизации $\left(L_{0}\right)$ кристалла при $T=0 \mathrm{~K}$ и $P=0$

$$
D_{b}=\frac{18 B_{0} V_{0}}{k_{n} a b N}=D_{s}+\Delta D, \quad D_{s}=\frac{L_{0}}{k_{n} / 2} .
$$

Для металлов эти две формулы дают одинаковый результат, а для ковалентных кристаллов глубина межатомного потенциала, восстановленная из модуля упругости $\left(D_{b}\right)$ приблизительно в два раза больше той величины, что следует из энергии атомизации кристалла $\left(D_{s}\right)$. Причем, разница: $\Delta D=D_{b}-D_{s}$, для кристаллов из элементов подгруппы углерода (алмаз, $\mathrm{Si}, \mathrm{Ge}$, $\alpha-\mathrm{Sn}, \mathrm{Pb})$ уменьшается с ростом массы атома $m$, и для свинца становится равной нулю: $\Delta D(\mathrm{~Pb})=0$. Энергия всей межатомной связи $\left(D_{b}\right)$ проявляется при упругой (обратимой) деформации, а энергия „слабой“ связи $\left(D_{s}\right)$ проявляется при пластической (необратимой) деформации ковалентного кристалла. Из значения $D_{b}$ необходимо рассчитывать такие параметры, при измерении которых не происходит разрыва межатомных связей: скорость звука, температура Дебая, коэффициент теплового расширения. При пластической (необратимой) деформации ковалентного кристалла - рвутся только „слабые“ звенья связи, и глубина потенциала (2) определяется величиной $D_{s}$. Поэтому из величины $D_{s}$ определяются такие (связанные с разрывом межатомных связей) параметры, как энергия сублимации, энергия активационных процессов (образования вакансий и самодиффузии), и удельная поверхностная энергия. Оба эти значения глубины потенциала (2) представлены в табл. 1: в первой строке $D_{b}$ - для упругой (обратимой) деформации, а во второй $D_{s}$ - для пластической (необратимой) деформации ковалентных кристаллов кремния и германия.

Используя указанные в табл. 1 параметры потенциала „упругого“ типа (т.е. с глубиной $D_{b}$ ), из выражений (10)-(12) получим значения, определяющие изменение параметров межатомного потенциала (2) с
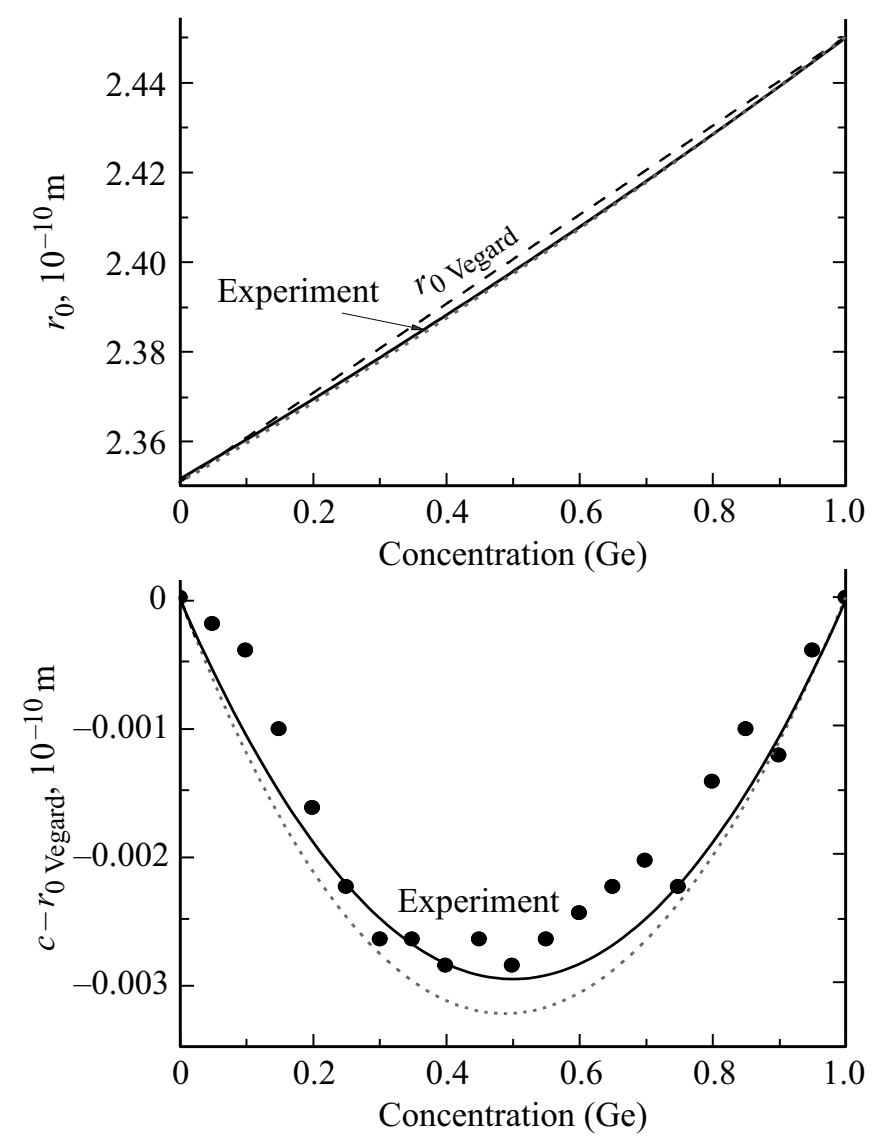

Рис. 1. Сверху - зависимость величины $c(C)$ от концентрации сплава $\mathrm{SiGe}$. На нижнем графике - разность $c(C)-r_{0 \text { Vegard }}(C)$. Точки и сплошная кривая - экспериментальные данные, точечная линия - результат наших расчетов. 

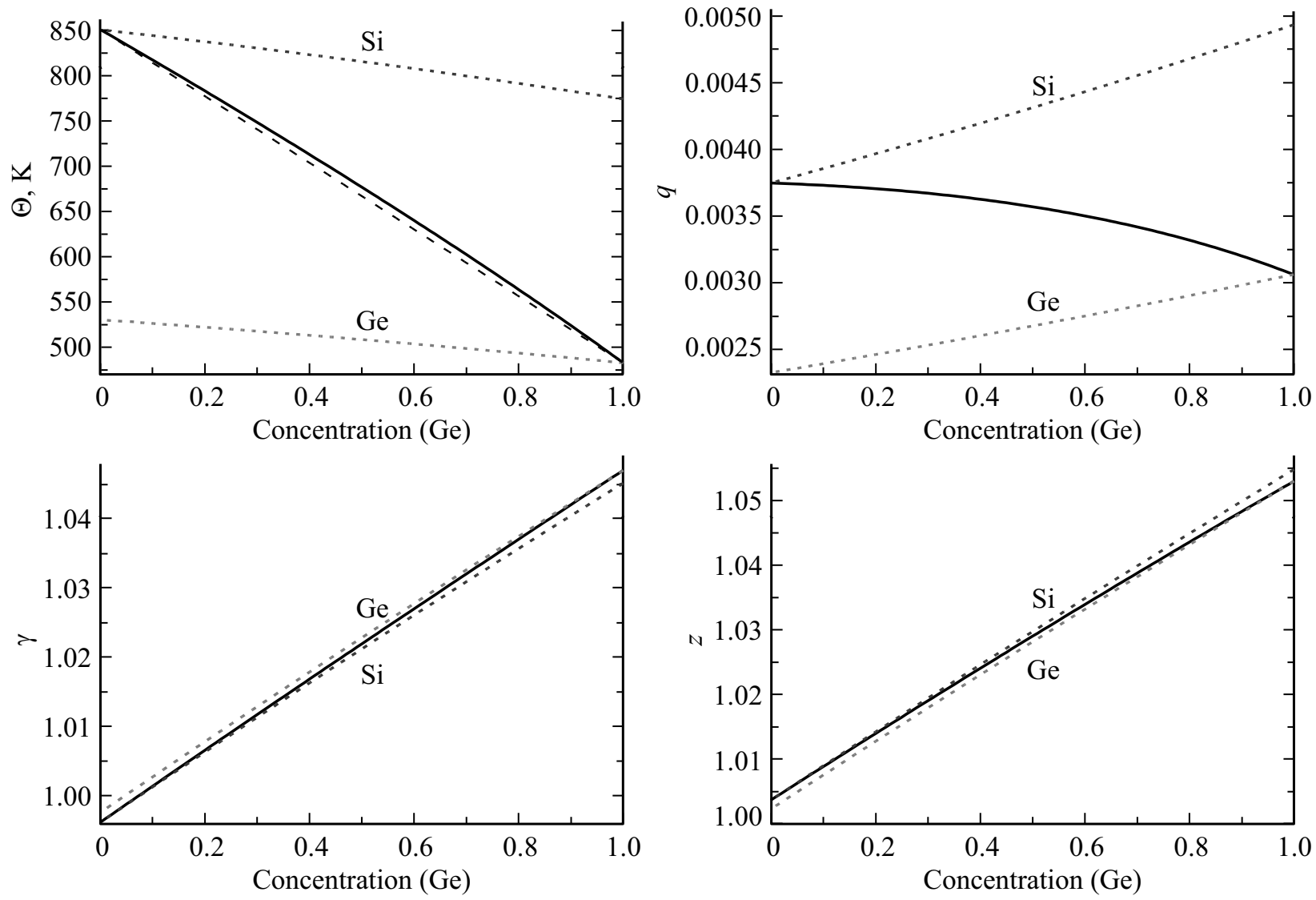

Рис. 2. Концентрационные зависимости (слева, сверху) для: температуры Дебая, первого (справа, сверху вниз), второго и третьего параметров Грюнайзена сплава $\mathrm{SiGe}$. Сплошной центральной кривой показана рассчитанная нами зависимость для общего параметра, точечными линиями показаны зависимости для парциальных параметров.

концентрацией $\mathrm{Ge}$ для сплава $\mathrm{Si}_{1-C} \mathrm{Ge}_{C}$ :

$$
\begin{gathered}
C_{N}=0.677024, \quad r_{0 \mathrm{SiGe}}=2.39196 \cdot 10^{-10} \mathrm{~m}, \\
D_{\mathrm{SiGe}} / k_{\mathrm{B}}=\left(D_{\mathrm{Si}} D_{\mathrm{Ge}}\right)^{1 / 2} / k_{\mathrm{B}}=54829.61 \mathrm{~K} \\
b_{\mathrm{SiGe}}=\left(b_{\mathrm{Si}}+b_{\mathrm{Ge}}\right) / 2=4.15 \\
a_{\mathrm{SiGe}}=\left(a_{\mathrm{Si}}+a_{\mathrm{Ge}}\right) / 2=2.615 .
\end{gathered}
$$

На рис. 1 вверху показана экспериментальная зависимость для $c(C)$ - расстояния между центрами ближайших атомов и для рассчитанного нами значения $r_{0}(C)$ - параметра координаты минимума парного потенциала (2) для сплава $\mathrm{Si}_{1-C} \mathrm{Ge}_{C}$. Верхняя пунктирная прямая - расчет по правилу Вегарда

$$
r_{0 \text { Vegard }} /\left[10^{-10} \mathrm{~m}\right]=2.351(1-C)+2.45 \cdot C .
$$

Сплошная кривая - это экспериментальная зависимость из [5]

$$
c_{\text {exper }} /\left[10^{-10} \mathrm{~m}\right]=2.35205+0.08661 \cdot C+0.0112 \cdot C^{2} .
$$

Эта зависимость определена из экспериментальных данных для параметра решетки $(l)$, который для струк- туры алмаза связан с кратчайшим межатомным расстоянием соотношением

$$
c=\left(3^{1 / 2} / 4\right) l=0.4330127 l .
$$

Нижняя точечная кривая, которая практически сливается с зависимостью (23), это результат наших расчетов для $r_{0}(C)$.

На рис. 1 внизу показана зависимость разности $c(C)-r_{0 \text { Vegard }}(C)$ от концентрации Ge: точки и сплошная кривая - экспериментальные данные из [5] и разность между зависимостями (22) и (23); точечная линия - результат наших расчетов. Из рис. 1 видно, что наша зависимость достаточно хорошо согласуются с экспериментальными данными, которые получены в [5] при $T=300 \mathrm{~K}$ с точностью $\pm 0.0005\left[10^{-10} \mathrm{~m}\right]$. Это позволяет утверждать, что отклонение от правила Вегарда обусловлено не только различной сжимаемостью решеток чистых компонент сплава (как это утверждалось в $[5,6])$, но и разным размером атомов, который определяет геометрические вероятности обнаружить атом в сплаве (6).

Отметим, что расчеты методом функционала плотности, проведенные в работе [6], показали плохое согласие с экспериментальными данными из [5]. Значение $|\Delta c(C)|$ 
Таблица 2. Рассчитанные значения температуры Дебая и параметров Грюнайзена при разной величине глубины межатомного потенциала для чистых кристаллов

\begin{tabular}{c|c|c|c|c|c|c|c|c|c|c}
\hline Кристалл & $\Theta, \mathrm{K}$ & $\Theta\left(m_{i}\right), \mathrm{K}$ & $\gamma$ & $\gamma\left(m_{i}\right)$ & $q \cdot 10^{3}$ & $q\left(m_{i}\right) \cdot 10^{3}$ & $z$ & $z\left(m_{i}\right)$ & $\Theta_{\text {exper }}[8]$ & $\gamma_{\text {exper }}[15]$ \\
\hline \multirow{2}{*}{$\mathrm{Si}$} & 850.185 & 773.823 & 0.9963 & 1.0451 & 3.747 & 4.932 & 1.0037 & 1.0549 & \multirow{2}{*}{$638-648.8$} & $1.00 \pm 0.03$ \\
& 549.058 & 535.789 & 0.9942 & 1.0429 & 5.802 & 7.124 & 1.0057 & 1.0570 & & \\
$\mathrm{Ge}$ & 482.222 & 529.619 & 1.0470 & 0.9977 & 3.063 & 2.328 & 1.0530 & 1.0023 & $373.3-374$ & $1.05 \pm 0.03$
\end{tabular}

Пр и меч ан и е. В первой строке показаны значения, полученные при $D_{b}$, т. е. для потенциала „упругого“ типа, а во второй строке - при $D_{s}$, т. е. для потенциала „пластичного“ типа. В двух правых столбцах показаны области разброса экспериментальных значений для температуры Дебая из обзора [8] и первого параметра Грюнайзена из [15].

в точке максимума, т. е. при $C(\mathrm{Ge})=0.5$ в [6] получилось очень большим: $\Delta c(C=0.5)=-0.039$, в то время как в [5] было получено: $\Delta c(C=0.5)=-0.00299 \pm 0.0005$, а в наших расчетах: $\Delta r_{0}(C=0.5)=-0.00324\left[10^{-10} \mathrm{~m}\right]$.

На рис. 2 показаны концентрационные зависимости для температуры Дебая: первого, второго и третьего параметров Грюнайзена сплава SiGe. Расчет выполнен по формулам (14) - (18) при использовании потенциала „упругого“ типа (т.е. с $\left.D_{b}\right)$ и при $c=r_{0}$, т.е. при $P=0$ и $T=0 \mathrm{~K}$.

На всех четырех графиках рис. 2 сплошной центральной кривой показана рассчитанная нами зависимость для общего параметра. Точечные линии - результат расчета парциальных значений параметра для виртуальной подрешетки с массой атома одного из компонент, но межатомное взаимодействие между атомами в которой описывается межатомным парным потенциалом (2) с параметрами зависящими от концентрации компонентов. На рис. 2 символом элемента указано, что данная зависимость относится к подрешетке данного компонента. На графике для температуры Дебая пунктирной прямой, лежащей рядом с зависимостью $\Theta(m)$, показана зависимость, рассчитанная как среднее арифметическое по концентрации от температур Дебая, вычисленных для чистых кристаллов:

$$
\Theta /[\mathrm{K}]=850.185(1-C)+482.222 \cdot C .
$$

Из рис. 2 видно, что зависимости общей температуры Дебая $\Theta(m)$ и параметров Грюнайзена от концентрации германия нелинейные. Это обусловлено как нелинейной зависимостью параметра $r_{0}(C)$ (см. рис. 1), так и нелинейной зависимостью других трех параметров парного потенциала (2) от концентрации Ge.

Отметим, что при использовании потенциала „пластичного“ типа (т.е. с глубиной $D_{s}$, которая приведена в нижних строках табл. 1), форма графиков будет аналогична тем, что показаны на рис. 2, но численные значения параметров изменятся. В табл. 2 представлены рассчитанные по формулам (14)-(18) значения температуры Дебая и параметров Грюнайзена при разной величине глубины межатомного потенциала для чистых кристаллов. В первой строке показаны значения, полученные при $D_{b}$, т.е. для потенциала „упругого“ типа, а во второй строке - при $D_{s}$, т. е. для потенциала „пластичного“ типа. Общие значения параметров рассчитаны для чистого кристалла. Эти значения соответствуют точкам на графиках рис. 2, где соединяются сплошная и пунктирная линии на вертикальной оси. Парциальные значения параметров (вида $\left.H\left(m_{i}\right)\right)$ рассчитаны для чистого кристалла $j$-го компонента со значением массы атома $i$-го компонента. Эти парциальные значения соответствуют точкам на графиках рис. 2, где пунктирная линия для $i$-го компонента достигает вертикальной оси, т. е. при $100 \%$ концентрации $j$-го компонента. В правых столбцах табл. 2 показаны области разброса экспериментальных значений для температуры Дебая из обзора $[8$, стр. 92] и для первого параметра Грюнайзена из [15] при $P=0$ и $T=300 \mathrm{~K}$. Легко видеть, что экспериментальные значения для чистых кристаллов укладываются в интервал между значениями $\Theta$ и $\gamma$, рассчитанными при $D_{b}$ и при $D_{s}$.

К сожалению, в литературе нет экспериментальных концентрационных зависимостей для температуры Дебая и параметров Грюнайзена для твердого раствора $\mathrm{SiGe}$. Поэтому представленные на рис. 2 рассчитанные результаты сравнивать не с чем. Но фазовая диаграмма (т.е. концентрационные зависимости для температуры солидуса и ликвидуса) сплава $\mathrm{SiGe}$, экспериментально изучена очень хорошо многими авторами и в общепринятом виде представлена в обзоре [12].

На рис. 3 показаны концентрационные зависимости для температуры солидуса и ликвидуса сплава $\mathrm{SiGe}$, рассчитанные при использовании потенциала „упругого“ типа. Заметим, что при переходе к потенциалу „Пластичного“ типа фазовая диаграмма практически не изменится. Это связано с тем, что входящее в формулы (20) и (21) отношение температуры Дебая в решетке раствора к парциальной температуре Дебая для подрешетки $i$-го компонента: $\Theta(m) / \Theta\left(m_{i}\right)$ для сплава $\mathrm{SiGe}$ не изменяются, ввиду малости энергии „нулевых колебаний“ по сравнению с глубиной межатомной связи для $\mathrm{Si}$ и $\mathrm{Ge}$

$$
\frac{k_{\mathrm{B}} A_{w}(m) \xi^{2}}{8 D} \ll 1, \quad \Theta(m) \cong\left[\frac{8 A_{w}(m) D}{k_{\mathrm{B}}}\right]^{1 / 2} .
$$




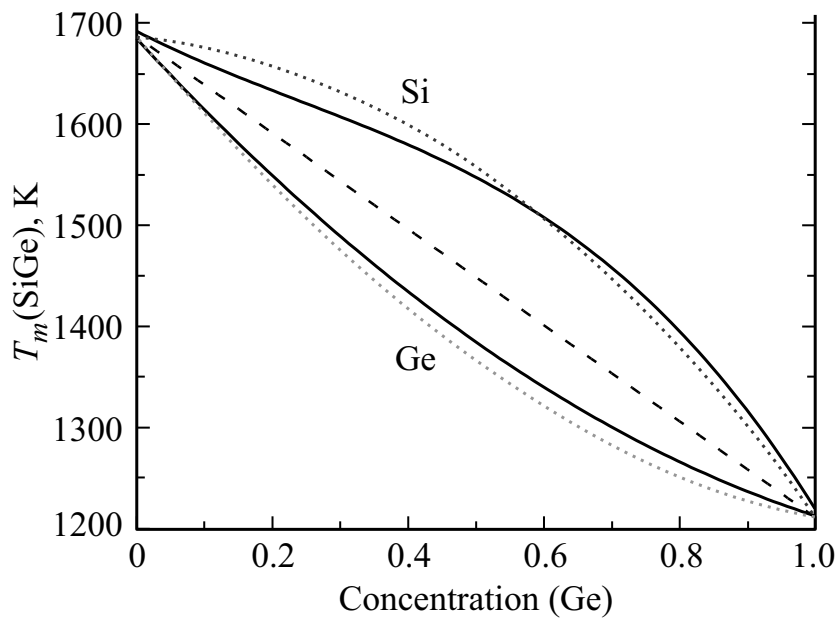

Pис. 3. Фазовая диаграмма сплава SiGe. Верхние выпуклые кривые - концентрационные зависимости для температуры ликвидуса, нижние вогнутые линии - концентрационные зависимости для температуры солидуса. Сплошные кривые экспериментальные зависимости из [12], точечные линии расчетные зависимости. Пунктирная прямая - расчет по формуле (26).

Сплошными кривыми на рис. 3 показаны экспериментальные зависимости из [12], имеющие следующий вид:

$$
\begin{gathered}
T_{s} /[\mathrm{K}]=1685.36071-728.64808 C+256.08582 C^{2}, \\
R_{\text {cor }}=0.9997 \\
T_{l} /[\mathrm{K}]=1692.57829-342.4064 C+343.75438 C^{2} \\
-474.08838 C^{3}, \quad R_{\text {cor }}=0.99814 .
\end{gathered}
$$

Данные зависимости получены путем аппроксимации экспериментальных данных из [12] указанными полиномами с коэффициентом достоверности $R_{c o r}$.

Пунктирные кривые получены из формул (20) и (21) для подрешеток германия - нижняя вогнутая кривая: $T_{s}(\mathrm{Ge})$, и кремния - верхняя выпуклая кривая: $T_{l}(\mathrm{Si})$, при использовании данных из табл. 1. При этом для концентрационной зависимости параметра Линдеманна, в соответствии с результатами [13], использовалась формула вида: $X_{L}(C)=0.136 P_{\mathrm{Si}}+0.1245 P_{\mathrm{Ge}}$, откуда следует:

$$
\begin{aligned}
& X_{L}(C) / X_{L \mathrm{Si}}=P_{\mathrm{Si}}+0.91544 P_{\mathrm{Ge}} \\
& X_{L}(C) / X_{L \mathrm{Ge}}=P_{\mathrm{Ge}}+1.09237 P_{\mathrm{Si}} .
\end{aligned}
$$

Пунктирной прямой на рис. 3 показана зависимость, рассчитанная как среднее арифметическое по концентрации от температур плавления чистых решеток $\mathrm{Si}$ и $\mathrm{Ge}$ :

$$
T_{m}(C) /[\mathrm{K}]=1211.45 C+1687.15(1-C) .
$$

Как видно из рис. 3, согласие рассчитанной фазовой диаграммы с экспериментальной вполне хорошее, что говорит о корректности как метода усреднения параметров парного межатомного потенциала, так и метода расчета общей и парциальной температуры Дебая и формул (20), (21).

В заключение отметим, что полученные результаты можно использовать во многих прикладных задачах. Например, используя зависимость для параметров парного межатомного взаимодействия от концентрации (соотношения (5), (6) и $(10)-(12))$ и зависимость для $m(C)$ в виде (13), можно с помощью методики из [14] рассчитывать изменение решеточных свойств твердого раствора с ростом давления.

\section{5. Об изменении фазовой диаграммы при уменьшении размера нанокристалла}

Методику также можно использовать для изучения эволюции фазовой диаграммы сплава замещения при уменьшении размера системы. Известно $[8,16]$, что при уменьшении размера кристалла (или числа атомов $N$ ) уменьшается среднее (по нанокристаллу) координационное число $k_{n}(N)$, причем уменьшение будет тем больше, чем заметнее форма нанокристалла отклонена от наиболее энергетически оптимальной формы (для RPмодели это куб). Это уменьшение $k_{n}(N)$, согласно (14) или (25), ведет к уменьшению температуры Дебая: $\Theta(N) \sim k_{n}(N)^{1 / 2}$, и, согласно (19), приводит к уменьшению температуры плавления: $T_{m}(N) \sim \Theta(N)^{2} \sim k_{n}(N)$. Применительно к нанокристаллу твердого раствора замещения это приведет к тому, что в формулах (20) и (21) с уменьшением размера будут уменьшаться только значения $T_{m \mathrm{~A}}$ и $T_{m \mathrm{~B}}$. Из этого следует, что разность между температурой ликвидуса и солидуса нанокристалла раствора будет уменьшаться при уменьшении его размера (т.е. числа атомов в нем) согласно зависимости

$$
\begin{aligned}
\Delta T_{l s}(C, N) & =T_{l}(C, N)-T_{s}(C, N) \\
& =k_{n}(N) \Delta T_{l s}(C, N=\infty) .
\end{aligned}
$$

Этот вывод о понижении значений $T_{m \mathrm{~A}}, T_{m \mathrm{~B}}$ и $\Delta T_{l s}$ при уменьшении размера нанокристалла согласуется с результатами, полученными более сложными методами для сплавов замещения $\mathrm{SiGe}$ в $[17]$ и $\mathrm{NiCu}$ в $[18,19]$.

Отметим, что как было показано в $[8,16]$ на примере RP-модели, при отклонении формы нанокристалла от наиболее оптимальной формы (для RP-модели это форма куба) функция $k_{n}(N)$ уменьшается с уменьшением $N$ сильнее. Поэтому при переходе к менее энергетически стабильным формам нанокристалла (стержневидные или пластинчатые) значения $T_{m \mathrm{~A}}, T_{m \mathrm{~B}}$ и $\Delta T_{l s}$ будут 
уменьшаться с уменьшением числа атомов $N$ более заметнее.

С другой стороны, как было показано в работах $[20,21]$ на примере чистых металлов и кремния, при некотором значении числа атомов $\left(N_{0}\right)$ теплота фазового перехода кристалл-жидкость (ФПК-Ж) исчезает: $h\left(N_{0}\right)=0$. В кластере такого $\left(N_{0}\right)$ размера ФПК-Ж уже невозможен. Из этого понятно, что для кластера из $N_{0}$ атомов (при любой концентрации компонент) классифицировать в каком фазовом состоянии (твердом или жидком) находится данный кластер невозможно, и для такого кластера выполняется: $\Delta T_{l s}\left(C, N_{0}\right)=0$. При этом в рамках RP-модели величина $N_{0}$ увеличивается при отклонении формы нанокристалла от наиболее оптимальной формы. Это также указывает на уменьшение величины $\Delta T_{l s}(C, N)$ с уменьшением числа атомов $N$ тем заметнее, чем более форма нанокристалла отклонена от наиболее оптимальной формы.

\section{6. Выводы}

Разработана методика получения концентрационной зависимости параметров парного межатомного потенциала для бинарного неупорядоченного твердого раствора замещения. Методика использует геометрическую вероятность обнаружения атома в растворе (которая учитывает разницу в размерах атомов компонент раствора), и учитывает отклонение межатомного расстояния раствора от правила Вегарда. На примере сплава $\mathrm{Si}_{1-C} \mathrm{Ge}_{C}$ показано, что отклонение зависимости $r_{0}(C)$ от правила Вегарда связано с различной сжимаемостью решеток чистых компонентов.

Разработана методика расчета концентрационной зависимости температуры Дебая и трех параметров Грюнайзена для бинарного сплава замещения, которая позволяет изучать как общие, так и парциальные значения данных свойств. На примере сплава $\mathrm{SiGe}$ показано, что изменение температуры Дебая и параметров Грюнайзена при изменении концентрации сплава происходит нелинейно.

Исходя из модели Линдеманна, получены формулы для расчета температуры солидуса и ликвидуса бинарного сплава замещения. На основе полученных зависимостей рассчитана фазовая диаграмма твердого раствора замещения $\mathrm{SiGe}$, и получено достаточно хорошее согласие с экспериментальными зависимостями как для температуры солидуса, так и для температуры ликвидуса.

Показано, что при уменьшении размера нанокристалла твердого раствора замещения разница между температурами ликвидуса и солидуса уменьшится тем больше, чем заметнее форма нанокристалла отклонена от наиболее энергетически оптимальной формы.

Автор благодарен Э.Н. Ахмедову, Н.Ш. Газановой, 3.М. Сурхаевой и М.М. Гаджиевой за помощь в работе и полезные дискуссии.

\section{Список литературы}

[1] http://www.ioffe.ru/SVA/NSM/Semicond/SiGe/basic.html

[2] T. Kumara Swamy, K.G. Subhadra, D.B. Sirdeshmukh. Pramana J. Phys. 43, 1, 33 (1994).

[3] Е.М. Соколовская, Л.С. Гузей. Металлохимия. Изд-во МГУ, М. (1986). 264 с.

[4] Дж. Гиршфельдер, Ч. Кертисс, Р. Берд. Молекулярная теория газов и жидкостей. ИЛ, М. (1961). 931 с. [J.O. Hirschfelder, Ch.F. Curtiss, B.B. Bird. Molecular Theory of Gases and Liquids. J. Wiley and Sons, N.Y. (1954). 1249 p.]

[5] J.P. Dismukes, L. Ekstrom, R.J. Paff. J. Phys. Chem. 68, 10, 3021 (1964).

[6] J.J. Pulikkotil, A. Chroneos, U. Schwingenschlögl. J. Appl. Phys. 110, 3, 036105 (2011).

[7] М.Н. Магомедов. ФТТ 45, 1, 33 (2003).

[8] М.Н. Магомедов. Изучение межатомного взаимодействия, образования вакансий и самодиффузии в кристаллах. Физматлит, М. (2010). 544 с.

[9] М.Н. Магомедов. ЖТФ 80, 9, 141 (2010).

[10] М.Н. Магомедов. Журн. неорган. химии 49, 12, 2057 (2004).

[11] С.М. Стишов. Письма в ЖЭТФ 71, 1, 25 (2000).

[12] R.W. Olesinski, G.J. Abbaschian. Bulletin Alloy Phase Diagrams 5, 2, 180 (1984).

[13] T. Soma. J. Phys. C 15, 9, 1873 (1982).

[14] М.Н. Магомедов. ФТТ 59, 6, 1065 (2017).

[15] А.Ф. Гончаров. Успехи физических наук 152, 2, 317 (1987).

[16] М.Н. Магомедов. Кристаллография 62, 3, 487 (2017).

[17] S. Bajaj, M.G. Haverty, R. Arroyave, W.A. Goddard III FRSC, S. Shankare. Nanoscale 7, 21, 9868 (2015).

[18] A.S. Shirinyan. Beilstein J. Nanotechnology 6, 1811 (2015).

[19] M.J. Cui, H. Lu, H. Jiang, Z. Cao, X. Meng. Sci. Rep. 7, 41990, 1 (2017).

[20] Q.S. Mei, K. Lu. Progr. Mater. Sci. 52, 8, 1175 (2007).

[21] М.Н. Магомедов. ЖТФ 86, 5, 92 (2016). 